\title{
Traveling chimera states for coupled pendula
}

\author{
Dawid Dudkowski ( Krzysztof Czołczyński • \\ Tomasz Kapitaniak
}

Received: 10 July 2018 / Accepted: 10 November 2018 / Published online: 20 November 2018

(C) The Author(s) 2018

\begin{abstract}
We investigate the phenomenon of traveling chimera states in the ring of self-excited coupled pendula suspended on the horizontally oscillating wheel. The bifurcation scenario of chimera creation and destruction is discussed, and the influence of the suspension's parameters on possible behavior is studied. We describe the properties of the investigated states, analyzing the traveling time as well as the dynamics of the pendula, depending on their position within different pattern's regions. The energy transfer method has been used to present how the units cooperate with each other, making the chimera arising possible. Unlike other studies on traveling chimera states, we examine the typical mechanical system including simple topology of coupling, which suggests that the described behavior can be observed naturally in the models of coupled dynamical oscillators.
\end{abstract}

Electronic supplementary material The online version of this article (https://doi.org/10.1007/s11071-018-4664-5) contains supplementary material, which is available to authorized users.

D. Dudkowski $(\varangle) \cdot$ K. Czołczyński · T. Kapitaniak Division of Dynamics, Lodz University of Technology, Stefanowskiego 1/15, 90-924 Lodz, Poland

e-mail: dawid.dudkowski@p.lodz.pl

K. Czołczyński

e-mail: krzysztof.czolczynski@p.lodz.pl

T. Kapitaniak

e-mail: tomasz.kapitaniak@p.lodz.pl
Keywords Chimera states $\cdot$ Coupled pendula $\cdot$ Energy balance

\section{Introduction}

The history of a surprising phenomenon called chimera state, which combines both coherent and incoherent patterns within one system's profile, is as short as rapid. For the first time, it was described by Kuramoto and Battogtokh in 2002 [1] and two years later confirmed by Abrams and Strogatz [2]. Nowadays, it constitutes a very wide and instantly developing branch of modern studies on nonlinear dynamics. Chimeric behavior can be found in a variety of models, among others Kuramoto networks [3], chemical oscillators [4], neural networks [5-8] or mechanical systems $[9,10]$. They have been observed for typical complex networks of oscillators [3-6,9-13], as well as for the small ones $[14,15]$, or even pure analytical [16]. Recent studies on chimera death for coupled chaotic oscillators can be found in [17], while in [18] the authors discuss the chimeric phenomenon in non-locally coupled excitable systems. The study on chimera states in twodimensional networks of the coupled oscillators was presented in [19]. The investigations on the creation process of these intriguing creatures show that in many cases they become a natural link between the complete coherence of the system and its pure chaotic dynamics [11-13]. Apart from theoretical investigations, chimera states were described for experimental setups of cou- 
pled systems [15,20,21]. A thorough review of chimera history and related research can be found in [22].

Among many new and interesting results, one of the very recently described is the so-called traveling chimera state. In this scenario, the chimeric pattern travels through consecutive oscillators arranged into a network, without any external cause, i.e., the movement is only controlled by the system itself. Traveling chimeras were observed for non-locally coupled phase oscillators [23], as well as in the models with hierarchical connectivities [24]. Recent studies on traveling patterns for neuronal networks including local synaptic gradient coupling can be found in $[25,26]$.

The importance of aforementioned papers [23-26] cannot be neglected, and their high contribution to the development of the research of chimeras' problems is obvious. However, it should be noted that in all these studies the authors investigate only theoretical models, with rather complex topology of coupling. In contrast, in this paper we investigate the traveling chimera state phenomenon for a simple network of self-excited pendula, which are coupled through typical scheme for mechanical systems. The dynamics has been determined directly from the Lagrange equations of motion. Due to the universal occurrence of the investigated model in nature, the obtained results are novel and allow us to understand better the chimeric behavior in general.

\section{Results}

Let us consider a ring of $N$ locally coupled selfexcited pendula suspended on the horizontally oscillating wheel, for which dynamics is given by Lagrange equations in the following form:

$$
\left\{\begin{array}{l}
\left(M+\sum_{j=1}^{N} m_{j}\right) r^{2} \ddot{\theta}+\sum_{j=1}^{N} m_{j} l_{j} r\left(\ddot{\varphi}_{j} \cos \varphi_{j}-\dot{\varphi}_{j}^{2} \sin \varphi_{j}\right) \\
+c r^{2} \dot{\theta}+k r^{2} \theta=0 \\
m_{i} l_{i} r \ddot{\theta} \cos \varphi_{i}+m_{i} l_{i}^{2} \ddot{\varphi}_{i}+c_{\varphi}\left(\mu \varphi_{i}^{2}-1\right) \dot{\varphi}_{i} \\
+m_{i} g l_{i} \sin \varphi_{i}+k_{s} l_{i}^{2}\left(\varphi_{i}-\varphi_{i-1}\right)+k_{s} l_{i}^{2}\left(\varphi_{i}-\varphi_{i+1}\right)=0
\end{array}\right.
$$

where $i=1, \ldots, N$, while variables $\theta$ and $\varphi_{i} \in$ $(-\pi, \pi]$ denote the angular position of the wheel and $i$ th pendulum, respectively. In our study, we fix the wheel's radius $r=1.0 \mathrm{~m}$ and stiffness $k=4.0$ $\mathrm{N} / \mathrm{m}$, pendula's masses $m_{i}=0.1 \mathrm{~kg}$ and lengths $l_{i}=0.24849 \mathrm{~m}$, van der Pol's damper parameters $c_{\varphi}=0.00499689 \mathrm{~kg} \mathrm{~m}^{2} / \mathrm{s}, \mu=32.88[27,28]$ (which

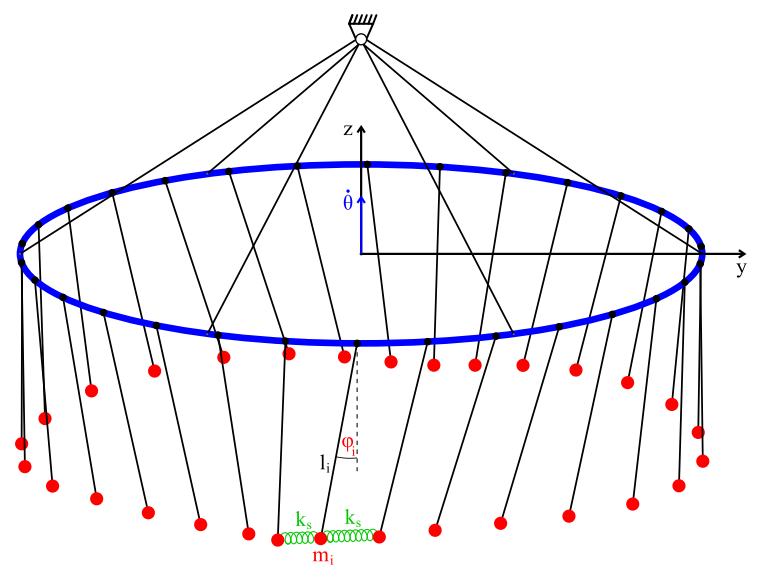

Fig. 1 Schematic of the investigated model (1) of coupled pendula suspended on the horizontally oscillating wheel. (Color figure online)

correspond to the amplitude and logarithmic decrement for a single, uncoupled pendulum at values $30^{\circ}$ $(0,35 \mathrm{rad})$ and $\ln (1.5)$, respectively) and standard gravity $g=9.81\left[\mathrm{~m} / \mathrm{s}^{2}\right]$. The pendula are coupled through elastic springs with stiffness $k_{s} \mathrm{~N} / \mathrm{m}$, which has been varied during the research along with the mass of the wheel $M(\mathrm{~kg})$ and its damping coefficient $c(\mathrm{Ns} / \mathrm{m})$.

Schematic of the system (1) is shown in Fig. 1. As shown, the wheel of mass $M$ (blue), hanged on the inextensible rods, can oscillate around $z$ axis. (Its angular position and velocity are denoted by $\theta$ and $\dot{\theta}$ variables, respectively.) The pendula of lengths $l_{i}$ (black lines) and masses $m_{i}$ (red dots) are suspended along the wheel. It should be noted that in our work we have considered planar pendula, i.e., the position of each unit in the phase space can be determined using one single variable $\varphi_{i}$. Each pendulum is locally coupled with its nearest neighbors by elastic springs of stiffness $k_{s}$ (green springs in Fig. 1-connections for only one unit have been shown for better clarity).

Note that the schematic shown in Fig. 1 only visualizes our model and does not include the results obtained during the research.

The investigations on the network (1) without coupling springs can be found in $[29,30]$.

In Fig. 2, one can observe the regions of different types of behavior of model (1) for $N=30$ pendula in two-parameter plane $\left(M, k_{S}\right)$. A bifurcation scenario for $M=1.25 \mathrm{~kg}$ (dashed vertical line in Fig. 2) is presented in Fig. 3. It should be noted that for each numerical step ( $M$ and $k_{s}$ values), the damping coefficient $c$ has been selected to preserve the fixed critical 


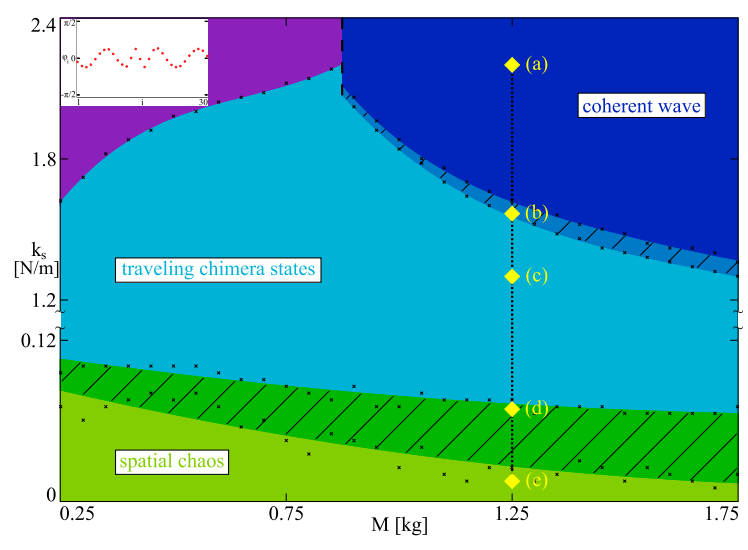

Fig. 2 Different types of dynamics of network (1) for varied wheel's mass $M$ and pendula coupling strength $k_{s}$. Each color represents possible behavior of the system, with hatched regions denoting transient responses. The examples of motion are shown in Fig. 3 and correspond to yellow squares marked on the dashed vertical line for $M=1.25 \mathrm{~kg}$. Network size $N=30$. (Color figure online)

damping $\zeta=c / 2 \sqrt{k\left(M+\sum_{j=1}^{N} m_{j}\right)}$, which in our calculations equals $\zeta=0.1975$. The borders of different regions have been smoothly approximated from numerical results, which are marked by small black crosses as shown in Fig. 2.

Beginning from the dark blue region in Fig. 2 within which the coupling is strong enough, the units form a typical continuous profile presented in the snapshot in the left panel in Fig. 3a for $k_{s}=2.2 \mathrm{~N} / \mathrm{m}$ (see movie M1 in Supplementary Material). As one can see, the pendula (marked by red dots) are displaced on a sinusoidal coherent wave (blue curve) and move along it with time. This type of behavior is typical for coupled oscillators and can be observed also in the models without moving suspension (opposed to our case).

In order to determine the moment of chimera creation (when decreasing coupling $k_{s}$ ), we have investigated the local-order parameter [31,32], which for each time iteration can be determined for a single $i$ th unit as $\lambda_{i}=\left|\frac{1}{2 \delta+1} \sum_{k=i-\delta}^{i+\delta} e^{j\left|\varphi_{i}-\varphi_{k}\right|}\right|$, where $j^{2}=-1$, while $\delta=1$. (Distance parameter has been chosen according to the topology of the coupling scheme.) The value of $\lambda_{i}=1$ corresponds to perfect synchronization with nearest neighbors. On the other hand, the more irregular the connection becomes, the more this parameter decreases.

By weakening the stiffness of the coupling springs, the coherent character of the network becomes less sta-
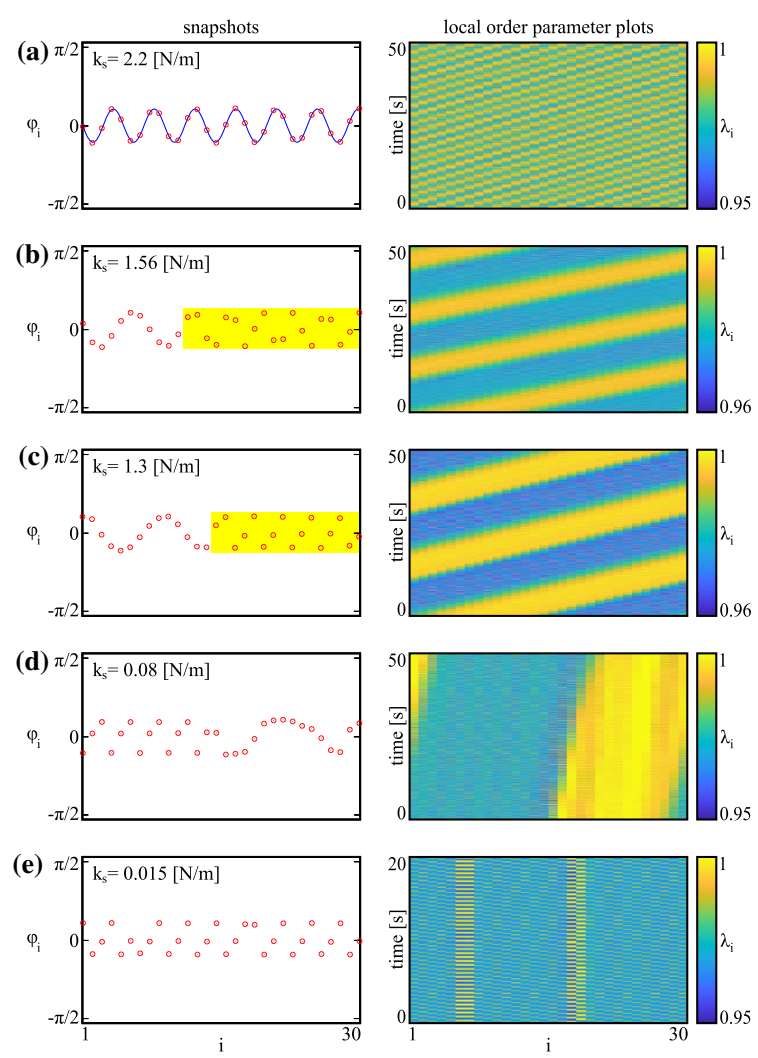

Fig.3 Typical bifurcation scenario for model (1) with fixed $M=$ $1.25 \mathrm{~kg}, c=1.6288 \mathrm{Ns} / \mathrm{m}$ and varied coupling strength $k_{s}$. In the left panel, the snapshots of pendula angular displacements are presented, while in the right one space-time plots of localorder parameter values are shown. From the top to the bottom, $k_{s}=2.2,1.56,1.3,0.08,0.015 \mathrm{~N} / \mathrm{m}$, respectively. (Color figure online)

ble and finally breaks down, creating a kind of a transient profile with coexisting wave and irregular motion of the part of the units. This type of dynamics is shown in Fig. $3 \mathrm{~b}$ for $k_{s}=1.56 \mathrm{~N} / \mathrm{m}$ (which corresponds to the hatched blue region in Fig. 2). The units belonging to the incoherent interval of the state are marked in yellow, and in the right panel in Fig. 3b, one can observe the stripes of regular $\left(\lambda_{i} \approx 1\right)$ and irregular $\left(\lambda_{i}<1\right)$ motion, determined by the values of the local-order parameter. The pattern is traveling with time, and each pendulum goes through both types of motion during system's movement.

Profile presented in Fig. $3 \mathrm{~b}$ can be identified as the chimera state with both temporal and spatial chaos behavior [11]. Indeed, one can observe that the rotating wave coexists with highly irregular pattern in the incoherent region, implying the chimeric nature of the network. However, further decrease in parameter $k_{s}$ 
allows us to observe another coexisting pattern which combines two typical responses related to the analyzed system. This type of behavior, denoted as 'traveling chimera states,' is shown in Fig. $3 \mathrm{c}\left(k_{s}=1.3 \mathrm{~N} / \mathrm{m}\right.$; see movie M2 in Supplementary Material) and exists in the light blue region in Fig. 2. The coherent wave from the previous example is still present (although with different length), but the profile of the pendula from the incoherent region (marked by yellow) has become more regular. Indeed, the units are placed in a strict order on three oscillating clusters.

Coexistence shown in Fig. 3c combines two natural types of dynamics for the discussed model. The rotating wave appears when the wheel is not moving, i.e., the energy between pendula is transferred only through the springs. On the other hand, the clusters are natural for the model without the coupling springs [29]. This type of dynamics is an example of a pure spatial chaos $[11,12]$, i.e., any combination of oscillators is possible, depending on the chosen initial conditions (when the springs are excluded). The appearance of the clusters within the chimera is strictly caused by the movements of the wheel on which the units are suspended. Note that the wheel oscillates with three times bigger frequency than each cluster and with each new cycle, it excites the clusters one by one. Moreover, due to the coupling springs, the order of the pendula within the structure is strictly determined and cannot be interrupted with variation of initial conditions. It should be noted that the chimera is still traveling. However, as the coupling decreases, the time of one full cycle increases. (This can be clearly observed for decreasing $k_{s}$-see Fig. 3d.)

The observed chimera pattern resides in a wide range of system's parameters (see the change of the scale on the vertical axis in Fig. 2), but when the coupling becomes small enough, the process of chimera destruction begins to occur. As shown in Fig. 3d, the coherent wave successively falls apart and with further decrease in $k_{s}$ splits into many parts. Moreover, the traveling velocity becomes very slow. This type of transient behavior (marked in Fig. 2 as green hatched region) can be observed until the strength of springs coupling pendula becomes irrelevant in the process of energy transfer between the units. When this occurs, only the pure spatial chaos is possible, marked in light green color in Fig. 2. The movement of oscillators is now induced only by the dynamics of the wheel, and the location of the units within the clusters strictly depends on the initial conditions. The example of this behavior is shown in Fig. 3e (see movie M3 in Supplementary Material). Any combination of the units is possible, similarly to the typical networks of oscillators coupled only through the wheel, without any topology of local or non-local coupling [29].

Note that we have observed also different types of coherent profile into which the traveling chimera states can bifurcate, i.e., the one shown as a snapshot in the violet region in Fig. 2. In this case, the previously described sinusoidal wave (Fig. 3a) splits into two waves of different lengths, which coexist within one network's profile. The rapid transition between these two types of scenarios lies around $M=0.875$ $\mathrm{kg}$ (marked by the dashed vertical line).

Further increase in the wheel's mass $M$ shows that the region of chimera existence shrinks and for the wheel which is heavy enough (i.e., almost not moving) it disappears. This implies that the oscillations of suspension are essential for the traveling chimera states to arise. On the other hand, for small values of $M$ a large number of perturbations and transitions between different scenarios of dynamics do not allow to determine the straight boundaries of typical examples of motion. Hence, the smallest value considered in our investigations equals $M=0.25 \mathrm{~kg}$.

The results presented in Figs. 2 and 3 have been obtained throughout the series of bifurcation analyses based on one, representative example of traveling chimera state, i.e., the profile shown in Fig. 3c. Indeed, starting from this state for each value of mass $M$ we have followed the solution up (increasing $k_{s}$ ) and down (decreasing $k_{s}$ ). The black crosses in Fig. 2, indicating the borders between regions, have been placed at the values for which we observe a substantial difference in the network profile (e.g., when the order in the clusters disappeared-transition from Fig. 3c to b).

Note that model (1) exhibits also different types of chimeric dynamics, which we have observed during our investigations. These have not been presented in this paper, as well as the study on possible coexistence between different scenarios.

The plots of local-order parameter for varying $k_{s}$ (right panel in Fig. 3b-d) show that depending on the coupling strength, the velocity of the chimera travel changes. In order to determine this dependency, we fix wheel's mass $M=1.25 \mathrm{~kg}$ and for the coupling values $k_{s} \in[0.08,1.6](\mathrm{N} / \mathrm{m})$ for which representative state from Fig. 3c exists, calculate the time of one full cycle of chimera travel, i.e., time after which the pendulum 

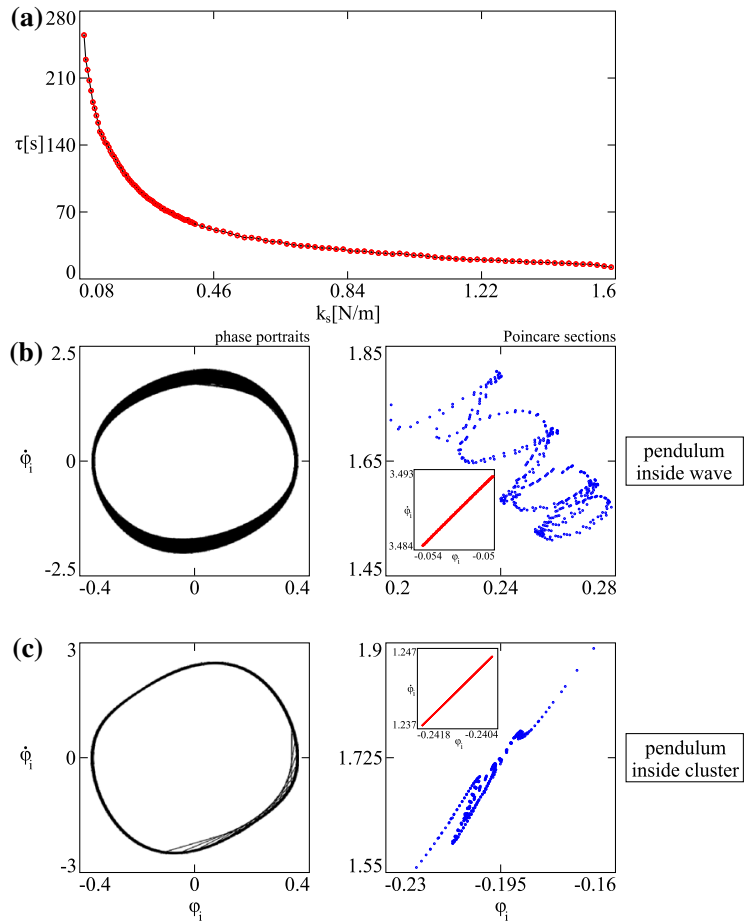

Fig. 4 In panel a the time of one full cycle of chimera travel (parameter $\tau$ ) is shown for fixed mass $M=1.25 \mathrm{~kg}$ and varying coupling strength $k_{s}$. The dynamics of the pendulum inside wave pattern (b) and cluster (c) is presented in the form of phase portraits and Poincare sections in left and right panels, respectively [parameters: $M=1.25 \mathrm{~kg}, k_{s}=0.2 \mathrm{~N} / \mathrm{m}$ ]. (Color figure online)

returns to its initial role in the pattern. The results can be observed in Fig. 4a, where in the vertical axis the travel time is shown (parameter $\tau$ ), while the stiffness of the springs $k_{s}$ is presented in the horizontal one. Numerical results (marked by red dots) exhibit a smooth, nonlinear dependence of time versus the coupling strength. Moreover, parameter $\tau$ monotonically decreases with an increase in $k_{s}$, i.e., when the elasticity of springs coupling pendula gets bigger, the chimera travels faster.

To study the dependence between the local dynamics of a particular pendulum and its position in the observed pattern, we fix parameters $M=1.25 \mathrm{~kg}, k_{s}=0.2 \mathrm{~N} / \mathrm{m}$ and plot the phase portraits and Poincare sections for a single $i$ th unit, when both $i$ th and $(i-1)$ th oscillators are within a wave or a cluster. The $(i-1)$ th pendulum has been chosen as the reference for the creation of Poincare maps. Indeed, when $\dot{\varphi}_{i-1}=0$ and $\varphi_{i-1}>0$ (which means that the $(i-1)$ th pendulum reaches its maximum), the position $\varphi_{i}$ and velocity $\dot{\varphi}_{i}$ of the $i$ th pendulum have been placed on the map. Since both pendula exhibit the same type of behavior while creating the Poincare sections (both belong to the wave or the cluster), the comparison of the maps can indicate the differences between the dynamics of the coexisting patterns.

The results are shown in Fig. 4b, c. As shown in Fig. $4 \mathrm{~b}$, when the oscillator belongs to the wave pattern, its dynamics is rather quasiperiodic. In the phase portrait (left panel), one can observe a typical twodimensional torus, while in the Poincare section (right panel), a complex structure reminds a fuzzy line segment. (The length of the $\varphi_{i}$ axis equals only 0.08.) We compare this map with the one calculated for the state from Fig. 3a (when the whole network is ordered only in the form of a sinusoidal wave), which is presented in the inbox in the right panel in Fig. 4b. As shown, the dynamics of the considered wave differs from the one observed for the state from Fig. 3a. On the other hand, for the pendulum inside a cluster domain (Fig. 4c) a different type of quasiperiodicity can be noticed, as shown in the phase portrait (left) and Poincare section (right). The map again has produced a line segment (even less fuzzy than the one in Fig. 4b). Moreover, it differs from the Poincare section for the network exhibiting only the cluster profile (e.g., as in Fig. 3e), which is shown in the inbox in the right panel in Fig. 4c. The fuzziness of the line segments in the maps can be caused by the traveling character of the chimera state-each pendulum jumps from one position into another within the cluster or wave domain.

The results presented in Fig. 4b, c exhibit that the traveling chimera states studied for system (1) combine two coexisting, most probably quasiperiodic, attractors and the dynamics of the pendulum strictly depends on its affiliation to wave or cluster pattern (which changes with time). These attractors differ from the ones observed for the cases of only sinusoidal (large $k_{s}$ ), or cluster (small $k_{s}$ ) behavior. Moreover, when the oscillator moves from one type of region into another one for a transient time its dynamics becomes highly irregular.

In order to study the possible mechanism behind the chimera traveling property, we have applied the energy balance method, which has been thoroughly discussed in [30]. The energy components for $i$ th pendulum arise from the equations of motion of network (1) (see [30] 

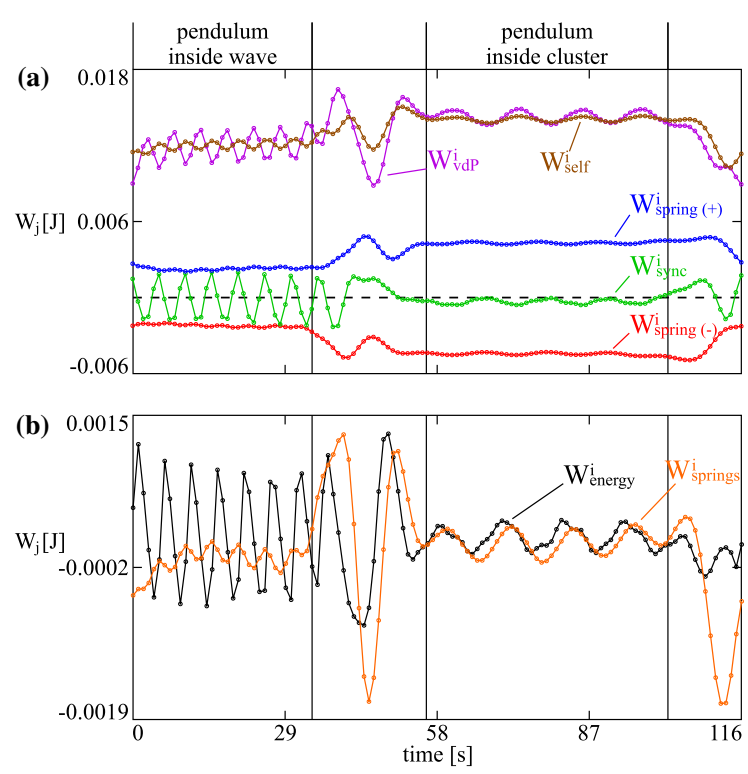

Fig. 5 Energy transfers for pendulum during one full cycle of chimera travel. In panel a, values of energy components [Eqs. (2)-(6), denoted by different colors] are shown, while in panel $\mathbf{b}$ increase/decrease in the total energy of oscillator (black) and energy produced by connecting springs (orange) is presented. Parameters: $M=0.75 \mathrm{~kg}, k_{s}=0.2 \mathrm{~N} / \mathrm{m}$. (Color figure online)

for details) and can be expressed as follows:

$$
\begin{aligned}
W_{\mathrm{self}}^{i} & =\int_{T} c_{\varphi} \dot{\varphi}_{i}^{2} \mathrm{~d} t, \\
W_{\mathrm{vdP}}^{i} & =\int_{T} c_{\varphi} \mu \varphi_{i}^{2} \dot{\varphi}_{i}^{2} \mathrm{~d} t, \\
W_{\text {sync }}^{i} & =\int_{T} m_{i} l_{i} r \ddot{\theta} \cos \varphi_{i} \dot{\varphi}_{i} \mathrm{~d} t, \\
W_{\text {spring(-) }}^{i} & =\int_{T} k_{s} l_{i}^{2}\left(\varphi_{i}-\varphi_{i-1}\right) \dot{\varphi}_{i} \mathrm{~d} t, \\
W_{\text {spring(+) }}^{i} & =\int_{T} k_{s} l_{i}^{2}\left(\varphi_{i}-\varphi_{i+1}\right) \dot{\varphi}_{i} \mathrm{~d} t,
\end{aligned}
$$

where (2) $W_{\text {self }}^{i}[\mathrm{~J}]\left((3) W_{\mathrm{vdP}}^{i}[\mathrm{~J}]\right)$ denotes the energy supplied (dissipated) by the van der Pol's damper, while (4) $W_{\text {sync }}^{i}[\mathrm{~J}]$ represents the energy transferred from the pendulum to the network via the wheel. The energy produced by elastic springs due to the coupling with $(i-1)$ th and $(i+1)$ th neighbor is given by (5) $W_{\text {spring(-) }}^{i}$ $[\mathrm{J}]$ and (6) $W_{\text {spring(+) }}^{i}(\mathrm{~J})$, respectively.

Moreover, in our study we consider:

$$
\begin{aligned}
W_{\mathrm{energy}}^{i}= & W_{\mathrm{self}}^{i}-W_{\mathrm{vdP}}^{i}-W_{\mathrm{sync}}^{i}-W_{\mathrm{spring}(-)}^{i} \\
& -W_{\text {spring }(+)}^{i}, \\
W_{\mathrm{springs}}^{i}=- & W_{\mathrm{spring(-)}}^{i}-W_{\text {spring }(+)}^{i}
\end{aligned}
$$

which represents the total increase/decrease in the energy of pendulum (7) and the energy transferred by the springs connected with unit (8). Note that the integrals given by (2)-(6) have been calculated for the time interval $T$ between two consecutive peaks of pendulum's position, which generally changes in time due to the traveling character of the chimera.

The transfer of energy during one full cycle of chimera travel is presented in Fig. 5, where the corresponding energy components and time are denoted on the vertical and the horizontal axes, respectively. The intervals of time within which the pendulum belongs to a wave or a cluster are marked with vertical lines, while the numerical results of partial energies (calculated for time between two consecutive pendulum's maximum peaks) are shown as colored dots.

As presented in Fig. 5, when the pendulum is inside the wave, the transfer of energy via the wheel varies, which can be detected by oscillating $W_{\text {sync }}^{i}$ term (green curve in Fig. 5a). However, the energies produced by left and right springs seem equal, and slight oscillations of total energy $W_{\text {springs }}^{i}$ marked by orange can be observed in Fig. 5b. Moreover, a clear difference between $W_{\text {springs }}^{i}$ (orange) and $W_{\text {energy }}^{i}$ (black) shown in Fig. 5b exhibits that only a small portion of pendulum's total energy is supplied by the springs. This situation changes when after a short, transient motion (accompanied by rapid fluctuations of energy terms) the oscillator gets inside the cluster domain. As presented in Fig. 5a, the magnitude of springs' energies has increased and there is almost no interaction between the unit and the suspension. Indeed, energy $W_{\text {sync }}^{i}$ (green) oscillates very close to zero (marked by hatched horizontal line). This fact is confirmed in Fig. 5b, where the plots of $W_{\text {energy }}^{i}$ and $W_{\text {springs }}^{i}$ terms are almost the same, with a slight shift. Moreover, the period of oscillations of energy $W_{\text {springs }}^{i}$ equals the time that the pendulum spends inside each of three-oscillator subclusters, of which the whole cluster pattern is built (see yellow region in Fig. 3c. These results clearly show that, in contrast to the previous case, the motion of the pendulum inside the cluster domain is mainly induced by the coupling with the nearest neighbors. This pattern continues through the whole cluster, which the oscillator finally leaves, getting back to the wave after some transient time. 


\section{Conclusions}

In conclusion, we have shown that depending on the system's parameters, traveling chimera states can be observed on the route from complete synchronization (wave profile) to the cluster patterns. In the considered case, the motion of a wheel on which the pendula are suspended is essential for chimeras to arise. Our results show that the discussed states consist of two coexisting attractors (one for the wave, and one for the cluster domain), and that the velocity of travel highly depends on the coupling strength between the pendula. On the other hand, energy transfer analysis gives an idea about the mechanism which stands behind the traveling property of the studied phenomenon. The network of the coupled pendula (1) considered in this paper includes a very simple topology of coupling, for which traveling chimera patterns have not been reported before, suggesting that this novel type of dynamics is robust and can arise in fundamental systems with rather simple coupling schemes, just to mention Josephson junctions, electronic circuits or chemical oscillators. Due to the simple character of the investigated model our numerical results outline an idea about possible experimental studies on traveling chimera states in the future.

Acknowledgements This work has been supported by the National Science Centre, Poland, MAESTRO ProgrammeProject No 2013/08/A/ST8/00/780. DD thanks the Foundation for Polish Science for financial support.

\section{Compliance with ethical standards}

Conflicts of interest The authors declare that they have no conflicts of interest.

Open Access This article is distributed under the terms of the Creative Commons Attribution 4.0 International License (http:// creativecommons.org/licenses/by/4.0/), which permits unrestricted use, distribution, and reproduction in any medium, provided you give appropriate credit to the original author(s) and the source, provide a link to the Creative Commons license, and indicate if changes were made.

\section{Appendix}

In Supplementary Material, we have shown typical patterns of the dynamics of the investigated model (1) in the form of animations. The description of the movies is as follows. In the upper part of each movie, the ani- mation of wheel (black dot) and pendula (color dots) motion are shown in the 2D plane, where on the horizontal axis the numbers of units are marked (with the wheel denoted by zero and the pendula by $1-30$ ), while in the vertical axis their displacements are given. These results correspond to the ones shown in the form of snapshots in Fig. 3 (left panel). On the other hand, in the lower part of each movie one can observe the animation of oscillating wheel (black rectangle with violet cross marker in the middle) with all suspended pendula marked by color dots. (The connections between the pendula have been omitted.) This animation presents the scheme from Fig. 1 when observing it from perpendicular direction to $(y, z)$ plane. (The wheel has been extended along $y$ axis, i.e., the very first pendulum on the LHS is coupled with the last one on the RHS.) It should be noted that the displacement of the wheel is multiplied by 100 for better clarity.

All animations have been produced using MATLAB software.

Movie M1: Typical coherent profile with pendula (red dots) displaced on a sinusoidal wave. The animation corresponds to Fig. 3a shown in the paper $\left(M=1.25, c=1.6288, k_{s}=\right.$ 2.2).

Movie M2: Traveling chimera state with coexisting wave and clusters profiles. Pendula belonging to the wave domain have been marked by red dots, while the ones in the clusters region have been shown as blue dots. As shown, the regions of different dynamics travel with time, involving consecutive oscillators. The animation corresponds to Fig. 3c shown in the paper $\left(M=1.25, c=1.6288, k_{s}=1.3\right)$.

Movie M3: Only coexisting clusters (pendula presented as blue dots). Animation corresponds to Fig. 3e shown in the paper $\left(M=1.25, c=1.6288, k_{s}=0.015\right)$.

\section{References}

1. Kuramoto, Y., Battogtokh, D.: Coexistence of coherence and incoherence in nonlocally coupled phase oscillators. Nonlinear Phenom. Complex Syst. 5, 380 (2002)

2. Abrams, D.M., Strogatz, S.H.: Chimera states for coupled oscillators. Phys. Rev. Lett. 93, 174102 (2004)

3. Laing, C.R.: The dynamics of chimera states in heterogeneous Kuramoto networks. Physica D 238, 1569 (2009) 
4. Tinsley, M.R., Nkomo, S., Showalter, K.: Chimera and phase-cluster states in populations of coupled chemical oscillators. Nat. Phys. 8, 662 (2012)

5. Xu, F., Zhang, J., Jin, M., Huang, S., Fang, T.: Chimera states and synchronization behavior in multilayer memristive neural networks. Nonlinear Dyn. 94, 775 (2018)

6. Tian, C., Cao, L., Bi, H., Xu, K., Liu, Z.: Chimera states in neuronal networks with time delay and electromagnetic induction. Nonlinear Dyn. 93, 1695 (2018)

7. Majhi, S., Ghosh, D.: Alternating chimeras in networks of ephaptically coupled bursting neurons. Chaos 28, 083113 (2018)

8. Bera, B.K., Ghosh, D., Lakshmanan, M.: Chimera states in bursting neurons. Phys. Rev. E 93, 012205 (2016)

9. Dudkowski, D., Maistrenko, Y., Kapitaniak, T.: Occurrence and stability of chimera states in coupled externally excited oscillators. Chaos 26, 116306 (2016)

10. Dai, Q., Liu, Q., Cheng, H., Li, H., Yang, J.: Chimera states in a bipartite network of phase oscillators. Nonlinear Dyn. 92, 741 (2018)

11. Dudkowski, D., Maistrenko, Y., Kapitaniak, T.: Different types of chimera states: an interplay between spatial and dynamical chaos. Phys. Rev. E 90, 032920 (2014)

12. Omelchenko, I., Maistrenko, Y., Hövel, P., Schöll, E.: Loss of coherence in dynamical networks: spatial chaos and chimera states. Phys. Rev. Lett. 106, 234102 (2011)

13. Jaros, P., Maistrenko, Y., Kapitaniak, T.: Chimera states on the route from coherence to rotating waves. Phys. Rev. E 91, 022907 (2015)

14. Maistrenko, Y., Brezetsky, S., Jaros, P., Levchenko, R., Kapitaniak, T.: Smallest chimera states. Phys. Rev. E 95, 010203 (2017)

15. Wojewoda, J., Czolczynski, K., Maistrenko, Y., Kapitaniak, T.: The smallest chimera state for coupled pendula. Sci. Rep. 6, 34329 (2016)

16. Abrams, D.M., Mirollo, R., Strogatz, S.H., Wiley, D.A.: Solvable Model for Chimera States of Coupled Oscillators. Phys. Rev. Lett. 101, 084103 (2008)

17. Xiao, G., Liu, W., Lan, Y., Xiao, J.: Stable amplitude chimera states and chimera death in repulsively coupled chaotic oscillators. Nonlinear Dyn. 93, 1047 (2018)

18. Dai, Q., Zhang, M., Cheng, H., Li, H., Xie, F., Yang, J.: From collective oscillation to chimera state in a nonlocally coupled excitable system. Nonlinear Dyn. 91, 1723 (2018)
19. Kundu, S., Majhi, S., Bera, B.K., Ghosh, D., Lakshmanan, M.: Chimera states in two-dimensional networks of locally coupled oscillators. Phys. Rev. E 97, 022201 (2018)

20. Martens, E.A., Thutupalli, S., Fourrière, A., Hallatschek, O.: Chimera states in mechanical oscillator networks. Proc. Natl. Acad. Sci. USA 110, 10563 (2013)

21. Kapitaniak, T., Kuzma, P., Wojewoda, J., Czolczynski, K., Maistrenko, Y.: Imperfect chimera states for coupled pendula. Sci. Rep. 4, 6379 (2014)

22. Panaggio, M.J., Abrams, D.M.: Chimera states: coexistence of coherence and incoherence in networks of coupled oscillators. Nonlinearity 28, R67 (2015)

23. Xie, J., Knobloch, E., Kao, H.-C.: Multicluster and traveling chimera states in nonlocal phase-coupled oscillators. Phys. Rev. E 90, 022919 (2014)

24. Hizanidis, J., Panagakou, E., Omelchenko, I., Schöll, E., Hövel, P., Provata, A.: Chimera states in population dynamics: networks with fragmented and hierarchical connectivities. Phys. Rev. E 92, 012915 (2015)

25. Bera, B.K., Ghosh, D., Banerjee, T.: Imperfect traveling chimera states induced by local synaptic gradient coupling. Phys. Rev. E 94, 012215 (2016)

26. Mishra, A., Saha, S., Ghosh, D., Osipov, G.V., Dana, S.K.: Traveling Chimera pattern in a neuronal network under local gap junctional and nonlocal chemical synaptic interactions. Opera Med. Physiol. 3, 14 (2017)

27. Guckenheimer, J.: Dynamics of the Van der Pol Equation. IEEE Trans. Circuits Syst. CAS-27, 11 (1980)

28. van der Pol, B.: The nonlinear theory of electric oscillations. Proc. Inst. Radio Eng. 22, 9 (1934)

29. Kapitaniak, M., Czolczynski, K., Perlikowski, P., Stefanski, A., Kapitaniak, T.: Synchronization of clocks. Phys. Rep. 517, 1 (2012)

30. Czolczynski, K., Perlikowski, P., Stefanski, A., Kapitaniak, T.: Why two clocks synchronize: energy balance of the synchronized clocks. Chaos 21, 023129 (2011)

31. Schroder, M., Timme, M., Witthaut, D.: A universal order parameter for synchrony in networks of limit cycle oscillators. Chaos 27, 073119 (2017)

32. Omelchenko, I., Provata, A., Hizanidis, J., Schöll, E., Hövel, P.: Robustness of chimera states for coupled FitzHughNagumo oscillators. Phys. Rev. E 91, 022917 (2015) 